\title{
Impact of high thoracic epidural anesthesia on incidence of perioperative atrial fibrillation in off-pump coronary bypass grafting: A prospective randomized study
}

Farhad Bakhtiary, MD, ${ }^{a}$ Panagiotis Therapidis, MD, ${ }^{a}$ Omer Dzemali, MD, ${ }^{a}$ Koray Ak, MD, Hanns Ackermann, MD, PhD, ${ }^{c}$ Dirk Meininger, MD, PhD, ${ }^{\mathrm{b}}$ Paul Kessler, MD, PhD, ${ }^{\mathrm{d}}$ Peter Kleine, MD, PhD, ${ }^{\text {a }}$ Anton Moritz, MD, PhD, ${ }^{\text {a }}$ Tayfun Aybek, MD, PhD, and Selami Dogan, MD ${ }^{a}$

From the Department of Thoracic \& Cardiovascular Surgery, ${ }^{a}$ Department of Anaesthesiology, Intensive Care Medicine and Pain Therapy, ${ }^{\mathrm{b}}$ and Department of Biomedical Statistics, ${ }^{c}$ Johann Wolfgang Goethe University Hospital, and the Department of Anesthesiology and Intensive Care Medicine, ${ }^{\mathrm{d}}$ Friedrichsheim Foundation, Frankfurt/ Main, Germany.

Received for publication Feb 22, 2007; revisions received March 15, 2007; accepted for publication March 29, 2007.

Address for reprints: Farhad Bakhtiary, MD, Department of Thoracic \& Cardiovascular Surgery, Johann Wolfgang Geothe University Hospital, Theodor-Stern-Kai 7, 60596 Frankfurt/Main, Germany (E-mail: farhad@ bakhtiary.de).

J Thorac Cardiovasc Surg 2007;134:460-4

$0022-5223 / \$ 32.00$

Copyright $\odot 2007$ by The American Association for Thoracic Surgery

doi:10.1016/j.jtcvs.2007.03.043
Objective: Atrial fibrillation is one of the most common complications in patients undergoing coronary artery bypass grafting. The goal of this study was to investigate the impact of high thoracic epidural anesthesia on reduction of perioperative arrhythmia in patients undergoing off-pump coronary artery bypass grafting.

Methods: We prospectively randomized 132 patients undergoing elective off-pump coronary bypass grafting using either general anesthesia $(\mathrm{GA})(\mathrm{n}=66)$ or combined general and high thoracic epidural anesthesia $(\mathrm{GA}+\mathrm{TEA})(\mathrm{n}=66)$. Incidence of perioperative arrhythmias such as atrial fibrillation, serum epinephrine levels, heart rate variability, and hemodynamic parameters were compared between groups.

Results: The incidence of perioperative dysarrhythmias was significantly lower $(P<.01)$ in the GA+TEA group $(3 \%)$ than in the GA group $(23.7 \%)$. Intraoperative sinus bradycardia occurred in $91 \%$ of the patients in the GA+TEA group versus $5.3 \%$ in the GA group. After induction of anesthesia, the mean systolic arterial pressure decreased significantly from $128 \pm 5$ to $92 \pm 4 \mathrm{~mm} \mathrm{Hg}$ and the heart rate from $74 \pm 9$ to $52 \pm 8$ beats $\cdot \min ^{-1}$ in the GA+TEA group, whereas in the GA group no significant hemodynamic changes were observed $(P<.001)$. Serum epinephrine levels were significantly lower in the GA+TEA group (69 \pm 11 to $35 \pm 7 \mathrm{ng} / \mathrm{dL})$ than in the GA group $(72 \pm 9$ to $70 \pm 9 \mathrm{ng} / \mathrm{dL})$.

Conclusions: In our study cohort, high thoracic epidural anesthesia in combination with general anesthesia reduced significantly the incidence of perioperative arrhythmias such as atrial fibrillation. Furthermore, we observed a significant reduction of epinephrine serum levels in this patient group. The results of this study support a combination of general anesthesia with thoracic epidural anesthesia as a multidisciplinary approach, which may lead to a better patient outcome, improvement of early analgesia, and reduction of perioperative complications in off-pump coronary artery bypass procedures. The potential risks of thoracic epidural anesthesia during off-pump coronary artery bypass procedures should not be underestimated.

$\mathrm{P}$ erioperative arrhythmias such as atrial fibrillation (AF) are the most common complications in patients undergoing off-pump coronary bypass grafting (OPCAB), with a reported prevalence of $5 \%$ to $29 \% .^{1-5}$ The etiology of AF is not widely known and has been related to many risk factors. ${ }^{6-8}$ Activation of the autonomic nervous system seems to enhance or trigger the initiation and perpetuation of AF. ${ }^{9}$ Recent studies have also reported that prophylactic administration of $\beta$-blocker therapy may reduce the incidence of postoperative AF and reduce the length of hospital stay after cardiac surgery. ${ }^{10,11}$

General anesthesia (GA) is the most commonly used anesthetic technique and is considered the "gold standard" for coronary artery bypass grafting (CABG) per- 


$$
\begin{aligned}
& \text { Abbreviations and Acronyms } \\
& \begin{aligned}
\mathrm{AF} & =\text { atrial fibrillation } \\
\mathrm{CABG} & =\text { coronary artery bypass grafting } \\
\mathrm{ECG} & =\text { electrocardiogram } \\
\mathrm{GA} & =\text { general anesthesia } \\
\mathrm{OPCAB} & =\text { off-pump coronary artery bypass grafting } \\
\mathrm{TEA} & =\text { high thoracic epidural anesthesia }
\end{aligned}
\end{aligned}
$$

formed either on pump or off pump. ${ }^{12}$ Within the past few years, however, high thoracic epidural anesthesia (TEA) as an adjunct to GA has become more prevalent and has been shown to be potentially beneficial in patients with coronary artery disease. ${ }^{13}$ Potential advantages of TEA include thoracic sympathicolysis with subsequent improvement of coronary perfusion, decreased heart rate, the latter being particularly important in OPCAB, decreased endogenous stress response, and a reduced risk for preoperative myocardial ischemia. Furthermore, improved postoperative pulmonary and gastrointestinal function with concomitant decreases in morbidity and mortality has been reported. ${ }^{12}$ Additionally, postoperative pain management is facilitated by continuous epidural application of analgesics. Such effective pain management improves postoperative mobilization and recovery. ${ }^{12}$ The risks associated with TEA are infections and hematoma formation with subsequent adverse neurologic sequelae. ${ }^{13}$ The aim of the present trial was to verify in a prospective randomized design whether the use of TEA leads to a reduction of perioperative arrhythmias in patients undergoing $\mathrm{OPCAB}$.

\section{Materials and Methods}

After approval by the institutional ethics board and with written informed consent, a total of 132 patients with symptomatic coronary artery disease were prospective enrolled in this study. All patients underwent elective OPCAB surgery and were randomized to receive either GA or combined GA+TEA. Patients with a history of atrial arrhythmias, those undergoing emergency operations, and patients requiring intraoperative inotropic support were excluded from this study. The incidence of perioperative arrhythmias such as AF, serum epinephrine levels, heart rate variability, and hemodynamic parameters were compared between the two groups. The hypothesis of the study was to determine whether the impact of the TEA in reduction of perioperative dysarrhythmias could be demonstrated in patients undergoing OPCAB.

\section{Surgical Technique}

The chest was opened either by complete sternotomy for double or triple $\mathrm{CABG}$ or by partial lower ministernotomy for single CABG. A small pediatric thorax retractor (Aeskulap, Tüttlingen, Germany) was used for exposition and harvesting of the left internal thoracic artery. Intravenous heparin was given in a standard dose of 150 IU/kg after thoracic artery dissection. A $75 \%$ reversal of heparin was performed with intravenous protamine at thorax closure. The activated coagulation time was measured to ensure appropriate heparin effect and reversal. An activated clotting time around 250 seconds during the operation was attained. After creating a pericardial cradle, the surgeon exposed the target vessels and used Octopus 4 (Medtronic Inc, Minneapolis, Minn) for stabilization of the target vessels. Anastomoses were performed in standard beating heart bypass technique using proximal control of the target coronary artery with a vessel loop and a blower mister to clear the anastomotic site.

\section{GA and TEA}

Antiplatelet therapy was stopped 5 days before the operation in all cases. In the TEA group, a multiport epidural catheter (Perifix Soft 505; B. Braun Melsungen AG, Melsungen, Germany) was inserted at the $\mathrm{T} 1 / 2$ or $\mathrm{T} 2 / 3$ intercostal space on the day before the operation. On the day of the operation, all patients were premedicated with oral midazolam, $7.5 \mathrm{mg}$.

On arrival of the patient in the preoperative holding area, intravenous access and direct blood pressure monitoring by catheterization of the radial artery were established regardless of the anesthetic technique used. In patients receiving TEA, a continuous epidural infusion with ropivacaine $0.16 \%$ and sufentanil $1 \mu \mathrm{g} \cdot \mathrm{mL}^{-1}$ at an hourly rate of 2 to $5 \mathrm{~mL}$ was started after a bolus dose of $6 \mathrm{~mL}$ to provide intraoperative analgesia.

After induction of general anesthesia, a central venous line was inserted and correct placement confirmed by intra-atrial electrocardiographic (ECG) lead (Alphacard; B.Braun AG, Melsungen, Germany). Additional monitoring consisted of continuous automated ST-segment analysis at $\mathrm{J}+60 \mathrm{~ms}$ for leads I, II, and $\mathrm{V}_{5}$ (Hellige Marquette Solar 8000 Patient Monitor; Marquette Medical Systems, Milwaukee, Wis). An ST-segment alteration of $1 \mathrm{~mm}$ or more $(0.1 \mathrm{mV})$ persisting more than 60 seconds was considered a significant alteration from baseline. Oxygenation and ventilation were continuously monitored by pulse oximetry and capnography. Body temperature was maintained in all patients with a warming blanket (Bair Hugger model 505; Augustine Medical, Inc, Eden Prairie, Minn) and continuously monitored with a rectal temperature probe.

Anesthesia was induced with propofol $\left(1.5 \mathrm{mg} \cdot \mathrm{kg}^{-1}\right)$ and remifentanil $\left(1 \mu \mathrm{g} \cdot \mathrm{kg}^{-1}\right)$ administered over 120 seconds. After loss of eyelash reflex, $0.1 \mathrm{mg} \cdot \mathrm{kg}^{-1}$ of cisatracurium was administered to facilitate tracheal intubation. Anesthesia was maintained with continuous infusion of propofol $\left(50-100 \mu \mathrm{g} \cdot \mathrm{kg}^{-1} \cdot \mathrm{min}^{-1}\right)$ and remifentanil $\left(0.1-0.3 \mu \mathrm{g} \cdot \mathrm{kg}^{-1} \cdot \mathrm{min}^{-1}\right)$. Positive-pressure ventilation with oxygen $50 \%$ in air was used. Tidal volume (8-10 $\left.\mathrm{mL} \cdot \mathrm{kg}^{-1}\right)$ and respiratory rate $\left(10-12 \mathrm{~min}^{-1}\right)$ were adjusted according to the end-tidal pressure of carbon dioxide to achieve normal ventilation (end-tidal pressure of carbon dioxide $35-40 \mathrm{~mm} \mathrm{Hg}$ ). Patients undergoing GA without TEA received intravenous metamizole (Novalgin; Aventis Pharma, Bad Soden, Germany), a peripheral analgesic derived from pyrazolone acid, $15 \mathrm{mg} \cdot \mathrm{kg}^{-1}$, before skin incision. Intravenous piritramide, a $\mu$-receptor agonist with a potency of 0.7 compared with morphine, $0.1 \mathrm{mg} \cdot \mathrm{kg}^{-1}$, was administered after completion of coronary anastomosis and repeated during wound closure. The thoracic epidural catheter was used for not only intraoperative but also postoperative pain management for 3 days. Depending on pain perception, patients re- 
TABLE 1. Patient characteristics

\begin{tabular}{lccc}
\hline & GA & GA+TEA & $P$ value \\
\hline $\mathrm{N}$ & 66 & 66 & \\
Age (y) & $64 \pm 9$ & $66 \pm 8$ & $\mathrm{NS}$ \\
Male & 58 & 54 & $\mathrm{NS}$ \\
Renal insufficiency & 32 & 25 & $\mathrm{NS}$ \\
Peripheral arterial disease & 21 & 18 & $\mathrm{NS}$ \\
Cerebrovascular disease & 14 & 17 & $\mathrm{NS}$ \\
EuroSCORE & $6.2 \pm 4$ & $5.4 \pm 6$ & $\mathrm{NS}$ \\
Sinus rhythm & 66 & 66 & $\mathrm{NS}$ \\
Preoperative $\beta$-blocker & 32 & 35 & $\mathrm{NS}$ \\
LVEF & & & \\
$\quad \geq 55 \%$ & 46 & 48 & $\mathrm{NS}$ \\
$\quad 35 \%-55 \%$ & 20 & 18 & $\mathrm{NS}$ \\
Previous MI & 37 & 36 & $\mathrm{NS}$ \\
Left main disease & 34 & 33 & $\mathrm{NS}$ \\
\hline
\end{tabular}

$G A$, General anesthesia; TEA, high thoracic epidural anesthesia; $N S$, not significant; $L V E F$, left ventricular ejection fraction; $M I$, myocardial infarction.

ceived additional analgesics and sedative hypnotic agents, as routinely used in our department.

Monitoring included arterial and central venous blood pressure measurement, ECG (leads II, aVF, and $\mathrm{V}_{5}$ ), pulse oximetry, and end-tidal carbon dioxide (Hellige Marquette Solar 8000 Patient Monitor, Marquette Medical Systems, Milwaukee, WI). Any newly developed sustained episode of cardiac arrhythmia was detected and printed with a continuous monitoring ECG system (Hellige Marquette Solar 8000 Patient Monitor; Marquette Medical Systems, Milwaukee, Wis) with automatic arrhythmia analysis for 48 hours. Thereafter, twice daily 12-lead ECGs were performed until hospital discharge and in each case with clinical symptoms caused by rhythm disturbance. In the case of documented arrhythmia, continuous ECG monitoring was restarted. AF was defined an episode of atrial fibrillation or flutter lasting for more than 30 seconds.

\section{Statistical Analysis}

Data were compiled and analyzed with Microsoft Excel (Redmond, Wash) and StatView (SAS Institute, Inc, Cary, NC). The baseline characteristics and hospital outcomes for both groups were compared by $\chi^{2}$ contingency or the Fisher exact test for categorical data and the Mann-Whitney $U$ test for continuous variables. Results are reported as the mean \pm standard deviation in text and tables.

\section{Results}

There were no significant differences in preoperative clinical characteristics including gender, sinus rhythm, left ventricular ejection fraction, preoperative antiarrhythmic medication, and cardiovascular risk factors between the two groups (Table 1).

Table 2 demonstrates operative data, incidence of arrhythmias, and the time of onset of perioperative AF. There was no significant difference between the operation time,
TABLE 2. Perioperative data

\begin{tabular}{lccc}
\hline & GA & GA+TEA & $\boldsymbol{P}$ value \\
\hline Operative time (min) & $121 \pm 45$ & $108 \pm 54$ & NS \\
No. of distal anastomoses & $2.3 \pm 1.1$ & $2.0 \pm 1.0$ & NS \\
Blood loss (mL) & $420 \pm 232$ & $380 \pm 208$ & NS \\
Ventilation time (h) & $7.0 \pm 4.2$ & $6.0 \pm 2.3$ & NS \\
AF & 18 & 2 & $<.01$ \\
Ventricular extrasystoles (counts) & 18 & 2 & $<.01$ \\
Sinus tachycardia & 32 & 6 & $<.01$ \\
Sinus bradycardia & 4 & 60 & $<.01$ \\
Ventricular fibrillation & 1 & 1 & NS \\
Time of onset of AF (d) & $2.0 \pm 1.4$ & $1.9 \pm 1.3$ & NS
\end{tabular}

$\overline{G A}$, General anesthesia; TEA, high thoracic epidural anesthesia; NS, not significant; $A F$, atrial fibrillation.

blood loss, ventilation time, and numbers of distal anastomoses. No relevant TEA-related complications such as puncture site infection, epidural hematoma, nerve injury, neurologic deficits, accidental dura puncture, or lower-limb motor block were observed. However, women, especially, were more prone to nausea and vomiting in the GA+TEA group. There was no in-hospital mortality in either group. The incidence of perioperative AF was significantly lower $(P<.01)$ in the GA+TEA group $(3 \%)$ than in the GA group (23.7\%). Time of onset of AF did not differ between the two groups. Patients with AF lasting more than 10 minutes or those requiring medical attention because of hemodynamic instability were treated with $\beta$-blockers (metoprolol $100 \mathrm{mg} / \mathrm{d}$ ), amiodarone $(5 \mathrm{mg} / \mathrm{kg}$ over $60 \mathrm{~min}$ utes followed by infusion of $1200 \mathrm{mg} / \mathrm{d}$ ), or digitoxin and electrical cardioversion (in case of hemodynamic instability or failure of pharmacologic treatment). Anticoagulation with heparin was started routinely. Intraoperative sinus bradycardia occurred in $91 \%$ of the patients in the GA+TEA group versus $5.3 \%$ in the GA group. After induction of anesthesia, the mean systolic arterial pressure decreased significantly from $128 \pm 5$ to $92 \pm 4 \mathrm{~mm} \mathrm{Hg}$ and heart rate from $74 \pm 9$ to $52 \pm 8$ beats $\cdot \mathrm{min}^{-1}$ in the GA+TEA group, whereas in the GA group no significant hemodynamic changes were observed (Figure $1 ; P<.001$ ). We observed one case of ventricular fibrillation in each group.

Serum epinephrine levels were significantly lower in group GA+TEA $\left(69 \pm 11\right.$ to $\left.35 \pm 7 \mathrm{ng} \cdot \mathrm{dL}^{-1}\right)$ than in in group GA (72 \pm 9 to $\left.70 \pm 9 \mathrm{ng} \cdot \mathrm{dL}^{-1} ; P<.001\right)$ (Figure 2 ).

The incidence of nonsustained ventricular extrasystole was significantly lower $(P<.01)$ in the GA+TEA group. There was no case of myocardial infraction in either group. Length of hospital stay did not differ between the two groups.

\section{Discussion}

Despite continuous development in minimizing surgical, anesthesiologic, or cardiopulmonary bypass trauma in CABG 


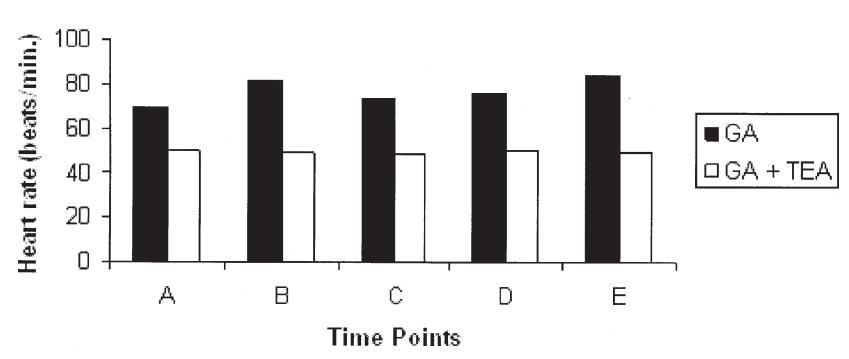

Figure 1. Intraoperative heart rate monitoring. $A$, Before induction of anesthesia; $B$, sternotomy; $C$, before anastomosis; $D$, after anastomosis; $E$, sternal closure. GA, General anesthesia; TEA, high thoracic epidural anesthesia.

procedures, perioperative morbidity and clinical complications are still significant. ${ }^{14} \mathrm{AF}$ remains one of the most frequent complications in patients undergoing $\mathrm{CABG}$, and it may lead to increased morbidity and length of hospital stay. Imbalance in the autonomic nervous system may cause changes in sinus node function and electrical atrial conduction as well as refractoriness previously shown to be related to the propensity for AF. Surgical trauma associated with increased sympathetic stimulation may also be a trigger for the onset of $\mathrm{AF}^{7,8}$

The current prospective randomized study was designed to investigate the impact of the TEA on the incidence of perioperative arrhythmias with major attention to AF. The perioperative use of GA in combination with TEA in our study cohort led to stress-response attenuation and intense perioperative analgesia. We demonstrated that patients in the GA+TEA group had a significantly lower incidence of $\mathrm{AF}$ and ventricular extrasystoles. In the GA+TEA group, serum levels of epinephrine were significantly lower, which may support the hypothesis in regard to preventing the imbalance of sympathetic activity.

TEA seems to attenuate the secretion of epinephrine from the adrenal gland, which may have a positive effect on the balance within the autonomous nervous system. The effect of TEA on heart rate and blood pressure is also in accordance with this observation. Inhibition of sympathetic activity during the perioperative period may reduce postoperative myocardial ischemia. ${ }^{15}$ This effect is most related to the balance of myocardial oxygen demand and supply. Most episodes of myocardial ischemia occur in the absence of major hemodynamic changes. ${ }^{16,17}$ Thus, the use of TEA in patients with significant coronary artery disease may improve oxygen supply, so long as blood pressure is maintained in a relatively normal range. These effects also facilitate beating heart surgery in regard to suturing conditions with a lower heart rate and blood pressure.

Our data support the hypothesis that perioperative imbalance of the autonomic nervous system may lead to a

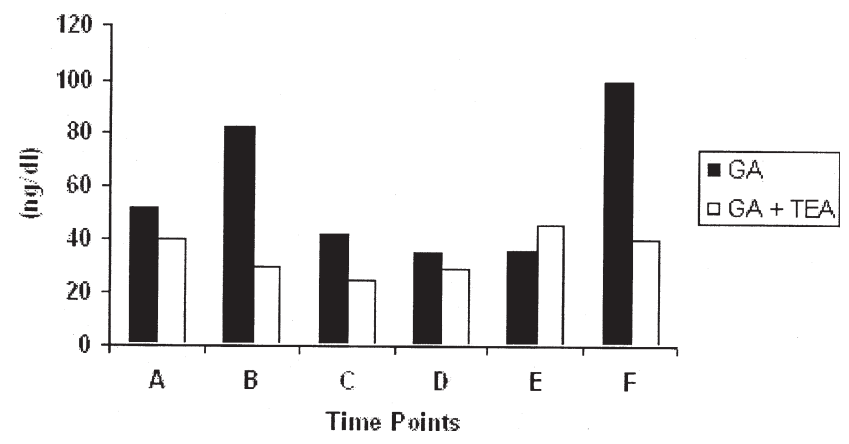

Figure 2. Serum epinephrine levels. $A$, Before induction of anesthesia; $B$, sternotomy; $C$, before anastomosis; $D$, after anastomosis; $E$, sternal closure; $F, 1$ hour postoperatively. GA, General anesthesia; TEA, high thoracic epidural anesthesia.

higher incidence of AF. We believe the reduced incidence of AF in our study cohort was related to sympathicolytic properties of TEA.

Furthermore, perioperative myocardial ischemia may be aggravated by sympathetic nerve activation, which disturbs the balance between coronary blood flow and myocardial oxygen demand. ${ }^{16,17}$

Recent studies have also shown that ropivacaine has a substantial anti-inflammatory effect. Blumenthal and associates ${ }^{18}$ could demonstrate that ropivacaine has strong antiinflammatory effects on neutrophils and endothelial cells both in vitro and in vivo.

In our study we could not measure any inflammatory parameters between the groups. However, we speculate that TEA may also decrease the systemic inflammatory response and consequently the development of AF.

\section{Study Limitations}

The study investigates a small number of patients. All patients were preselected by an anesthesiologist for eligibility to receive an epidural catheter before randomization. Patients with contraindications for TEA were excluded from the study. We did not measure any inflammatory parameters. Overall, longer follow-up periods and also a larger number of patients are necessary to corroborate our findings.

\section{References}

1. Aranki SF, Shaw DP, Adams DH, Rizzo RJ, Couper GS, VanderVliet $\mathrm{M}$, et al. Predictors of atrial fibrillation after coronary artery surgery. Current trends and impact on hospital resources. Circulation. 1996; 94:390-7.

2. Mathew JP, Parks R, Savino JS, Friedman AS, Koch C, Mangano DT, et al. Atrial fibrillation following coronary artery bypass graft surgery: predictors, outcomes, and resource utilization. MultiCenter Study of Perioperative Ischemia Research Group. JAMA. 1996;276:300-6.

3. Mueller XM, Tevaearai HT, Ruchat P, Stumpe F, von Segesser LK. Did the introduction of a minimally invasive technique change the incidence of atrial fibrillation after single internal thoracic artery-left 
anterior descending artery grafting? J Thorac Cardiovasc Surg. 2001;121:683-8.

4. Cohn WE, Sirois CA, Johnson RG. Atrial fibrillation after minimally invasive coronary artery bypass grafting: a retrospective, matched study. J Thorac Cardiovasc Surg. 1999;117:298-301.

5. Creswell LL, Schuessler RB, Rosenbloom M, Cox JL. Hazards of postoperative atrial arrhythmias. Ann Thorac Surg. 1993;56:539-49.

6. Stamou SC, Dangas G, Hill PC, Pfister AJ, Dullum MK, Boyce SW, et al. Atrial fibrillation after beating heart surgery. Am J Cardiol. 2000;86:64-7.

7. Scherer M, Sirat AS, Aybek T, Martens S, Kessler P, Moritz A. Thoracic epidural anesthesia does not influence the incidence of postoperative atrial fibrillation after beating heart surgery. Thorac Cardiovasc Surg. 2003;51:8-10.

8. Siebert J, Anisimowicz L, Lango R, Rogowski J, Pawlaczyk R, Brzezinski $\mathrm{M}$, et al. Atrial fibrillation after coronary artery bypass grafting: does the type of procedure influence the early postoperative incidence? Eur J Cardiothorac Surg. 2001;19:455-9.

9. Kalman JM, Munawar M, Howes LG, Louis WJ, Buxton BF, Gutteridge $\mathrm{G}$, et al. Atrial fibrillation after coronary artery bypass grafting is associated with sympathetic activation. Ann Thorac Surg. 1995;60: 1709-15.

10. Halonen J, Hakala T, Auvinen T, Karjalainen J, Turpeinen A, Uusaro $A$, et al. Intravenous administration of metoprolol is more effective than oral administration in the prevention of atrial fibrillation after cardiac surgery. Circulation. 2006;114(1 Suppl 1):I1-4.

11. Connolly SJ, Cybulsky I, Lamy A, Roberts RS, O'brien B, Carroll S, et al. Beta-Blocker Length Of Stay (BLOS) study. Double-blind, placebo-controlled, randomized trial of prophylactic metoprolol for reduction of hospital length of stay after heart surgery: the beta-Blocker Length Of Stay (BLOS) study. Am Heart J. 2003;145: 226-32.

12. Wattwil M, Sundberg A, Arvill A, Lennquist C. Circulatory changes during high thoracic epidural anaesthesia-influence of sympathetic block and of systemic effect of the local anaesthetic. Acta Anaesthesiol Scand. 1985;29:849-55.

13. Kessler P, Aybek T, Neidhart G, Dogan S, Lischke V, Bremerich DH, et al. Comparison of three anesthetic techniques for off-pump coronary artery bypass grafting: general anesthesia, combined general and high thoracic epidural anesthesia, or high thoracic epidural anesthesia alone. J Cardiothorac Vasc Anesth. 2005;19:32-9.

14. Roques F, Nashef SA, Michel P, Gauducheau E, de Vincentiis C, Baudet E, et al. Risk factors and outcome in European cardiac surgery: analysis of the EuroSCORE multinational database of 19,030 patients. Eur J Cardiothorac Surg. 1999;15:816-23.

15. Slogoff S, Keats AS. Randomized trial of primary anesthetic agents on outcome of coronary artery bypass operations. Anesthesiology. 1989;70: 179-88.

16. Liu S, Carpenter RL, Neal JM. Epidural anesthesia and analgesia. Their role in postoperative outcome. Anesthesiology. 1995;82:1474-506.

17. Slogoff S, Keats AS. Myocardial ischemia revisited. Anesthesiology. 2006;105:214-6.

18. Blumenthal S, Borgeat A, Pasch T, Reyes L, Booy C, Lambert M, et al. Ropivacaine decreases inflammation in experimental endotoxininduced lung injury. Anesthesiology. 2006;104:961-9. 